\title{
Capacitive Sensing of Narrow-Band ECG and Breathing Activity of Infants through Sleepwear
}

\author{
Akinori Ueno, Tatsuya Imai, Daisuke Kowada and Yoshihiro Yama \\ Tokyo Denki University \\ Japan
}

\section{Introduction}

Sudden infant death syndrome (SIDS) is defined as the sudden unexpected death of an infant $<1$ year of age, with onset of the fatal episode apparently occurring during sleep, that remains unexplained after a thorough investigation, including performance of a complete autopsy and review of the circumstances of death and the clinical history (Krous et al., 2004). SIDS has ranked the third leading cause of death for infants in Japan in 2007, after congenital malformations, deformations and chromosomal abnormalities, and certain conditions originating in the perinatal period (Statistics and Information Department, 2007). An apparent life threatening event (ALTE) is defined as an episode that is frightening to the observer and that is characterized by some combination of apnea (central or occasionally obstructive), color change (usually cyanotic or pallid), marked change in muscle tone, choking or gagging (Little et al., 1987). In order to prevent a recurrence of ALTE or to avoid an occurrence of SIDS, home monitoring of breathing activity and heart rate (HR) for infants may be introduced at the discretion of the doctor or the parent(s). In conventional monitors such as VitaGuard (GeTeMed GmbH, Germany) and SmartMonitor 2 (Children's Medical Ventures, USA), a conductive adhesive is used for maintaining reliable ohmic contact of electrodes with the skin. Therefore, monitoring for a long period of time using conventional methods may cause irritation and skin allergy. Besides, in some cases, adhesion of the paste was so tight for their skin that the skin was peeled off when the electrode was detached from the body surface after the long time monitoring. To relieve the potential of irritation and damage to the skin, Gramse et al. (Gramse et al., 2003) proposed special pajamas named MamaGoose (Verhaert Design and Development, Belgium), which incorporated dry electrodes and strain gauge for cardiopulmonary monitoring. Catrysse et al. (Catrysse et al., 2004) also addressed the similar problem by employing textile electrodes and a coil-shaped fabric sensor, which do not require any conductive adhesive for the measurement. The ideas of dry sensors embedded in clothing are quite rational. However, there are still some challenges to be addressed regarding direct contact of sensors with the skin, because that may provoke skin allergy and dermatitis. Moreover, repetitive use of the embedded electrode has a disadvantage in a hygiene standpoint in highly humid countries such as Japan, because they can't be washed easily. 
In order to obviate these risks and the disadvantage, our research group advanced the principle of capacitive sensing and succeeded in detecting electrocardiographic potential (ECG) through commonly available cloth from the subject's limb (Ueno et al., 2004), from the dorsal surface of adult subjects (Furusawa et al., 2003, Ueno et al., 2007a, and Ueno et al., $2007 \mathrm{~b}$ ) and from that of infants (Kato et al., 2006) in a supine position. This approach eliminated direct contact of the electrodes to the skin and then enabled the interjacent cloth being changed and washed handily. Moreover, with a view to application to preventing ALTE and SIDS, our group extended the capacitive sensing technique to that capable of measuring breathing activity simultaneously with ECG (Ueno \& Yama, 2008, and Yama \& Ueno, 2009). In this chapter, we describe the principle of the capacitive sensing technique and present our latest advances for these capacitive sensing approaches.

\section{Principle of Measurement}

\subsection{Principle of Capacitive Sensing of ECG}

The proposed approach of capacitive sensing is an expansion of the principle of the capacitive (or insulator) electrode (Richardson et al., 1968, and Lopez \& Richardson, 1969). Instead of rigid metal electrode and insulator in their coupling, the proposed coupling is composed of a conductive fabric electrode, clothes such as sleepwear and diaper, and the skin of the subject, as shown in Fig.1.
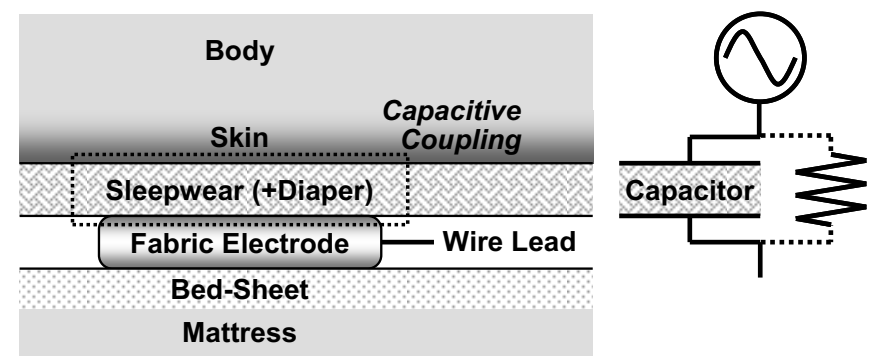

Fig. 1. A schematic model of the proposed capacitive coupling involving a fabric electrode, inserted clothes of sleepwear (plus diaper) and the skin, and its equivalent circuit elements

According to the equivalent circuit elements in Fig.1, impedance $Z[\Omega]$ of the coupling is given by

$$
Z=\frac{R}{\sqrt{1+(2 \pi f C R)^{2}}}=\frac{1}{\sqrt{1 / R^{2}+(2 \pi f C)^{2}}}
$$

where $C[F]$ is capacitance of the coupling, $R[\Omega]$ is resistance of the inserted clothes and $f$ $[\mathrm{Hz}]$ is frequency of the source signal. Since $R$ is so high in dry condition that it can be regarded as infinity, impedance of the coupling at dry condition $\left(Z_{R=\infty}\right)$ can be described as follows:

$$
Z_{R=\infty}=\frac{1}{2 \pi f C}
$$

Therefore, the coupling can carry an alternating bioelectric current through the capacitance of the coupling. Since direct contact of electrode with the skin is unnecessary in this 
approach, the proposed method can eliminate potential causes of metal allergy and dermatitis experienced in conventional methods. Moreover, the proposed method has an advantage in enabling commonly available clothes to be inserted between electrode and the skin. In equation (2), $C$ can be represented by the following equation (3) using coupling area $S\left[\mathrm{~m}^{2}\right]$, distance $d[\mathrm{~m}]$ between electrode and the skin, and permittivity $\varepsilon[\mathrm{F} / \mathrm{m}]$ of the mediated clothes.

$$
C=\varepsilon \frac{S}{d}
$$

By putting equation (3) into equation (2), $Z_{R=\infty}$ can be expressed as

$$
Z_{R=\infty}=\frac{1}{2 \pi} \times \frac{d}{f_{\varepsilon S}}
$$

Thus, the wider the electrode area becomes (or the closer the coupling distance becomes), the smaller the coupling impedance results. However, due to smaller permittivity of the commonly available clothes than that of insulators incorporated in commercial capacitors, coupling impedance in reality is estimated to reach in the order of a few or a few tens of Giga-ohms. Accordingly, commercial electrocardiographs having input impedance around a hundred Mega-ohms are incapable of detecting ECG signal through the clothes. In the proposed method, voltage loss at the coupling is reduced by employing an impedance transformer IC having high input impedance at the front end so as to achieve reasonable signal to noise ratio (SNR).

On the other hand, once the resistance of inserted clothes is decreased, for instance, by perspiration or moisture in the atmosphere, the following inequality expression is obtained:

$$
Z=\frac{1}{\sqrt{\frac{1}{R^{2}}+(2 \pi f C)^{2}}}<\frac{1}{2 \pi f C}\left(=Z_{R=\infty}\right)
$$

Thus, the more the subject sweats, the higher SNR of the output signal becomes. In other words, there is no adverse effect of perspiration of the subject on the SNR of the obtained signal in this approach.

\subsection{Principle of Breathing Activity Measurement}

In our previous experiments, it has been revealed that the capacitive sensing is susceptible to body motion of the subject. This susceptibility is assumed because the motion alters geometric parameters $S$ and $d$ of the coupling in equation (3), and thereby changes capacitance and impedance of the coupling. This disadvantage can be regarded as an advantage from the other side that the capacitive sensing is highly sensitive to body motion. In fact, some of the obtained signals had contained a periodic variation involving low frequency component and had seemed to be caused by breathing chest movement. Considering all these facts, a separation filter (Asaishi et al., 2002) shown Fig. 2 is employed in the proposed measuring circuit to divide the detected signal into a high frequency component including ECG and a low frequency component containing breathing activity. In order to design a differential separation filter with high common mode rejection ratio (CMRR), mirroring technique (Pallàs-Areny \& Webster, 1999) is applied to a single-ended separation filter.

Looking at a relationship between $v_{\text {in }}$ and $v_{\text {out_High }}$ of a single-ended filter in Fig. 2, $v_{\text {out_High }}$ is integrated with a time constant $\tau$ and then returned negatively to $v_{i n}$. Since the difference 
between $v_{\text {in }}$ and the integrated value of $v_{\text {out_High }}$ is amplified with an amplification factor $A$ to obtain $v_{\text {out_High }}$, transfer function $G_{H i g h}(s)$ between $v_{\text {in }}$ and $v_{\text {out_High }}$ is given by

$$
G_{\text {High }}(s)=\frac{v_{\text {out } \_ \text {High }}}{v_{\text {in }}}=\frac{\tau s}{1+\frac{\tau}{A} s}
$$

Equation (6) represents a trasfer function of 1st-order high-pass Butterworth filter. On the other hand, $v_{\text {out_Low }}$ is produced by the integration of $v_{\text {out_High }}$ and then transfer function $G_{\text {Low }}(s)$ between $v_{\text {in }}$ and $v_{\text {out } L \text { Low }}$ is

$$
G_{\text {Low }}(s)=G_{H i g h}(s) \cdot \frac{1}{\tau s}=\frac{1}{1+\frac{\tau}{A} s}
$$

Equation (7) means a transfer function of 1st-order low-pass Butterworth filter. In the present study, the amplification factor $A$ and the time constat $\tau$ are set to $1 \mathrm{v} / \mathrm{v}$ and $0.16 \mathrm{sec}$ respectively, so as to achieve a corner frequency $f_{\mathrm{c}}$ of $1 \mathrm{~Hz}$.

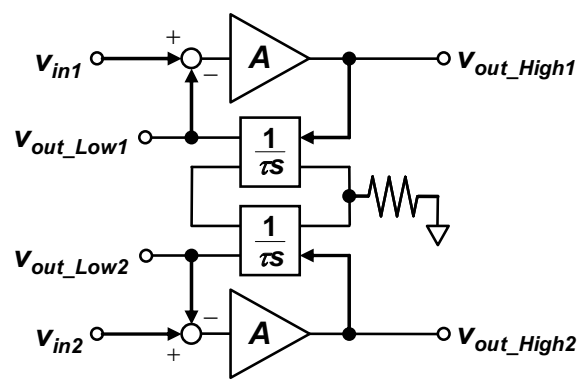

Fig. 2. A block diagram of the differential separation filter

\section{Materials and Methods}

\subsection{Bed-sheet Electrode Unit}

Both ECG and breathing activity signals were picked up by a common bed-sheet electrode unit placed on a mattress. The unit was composed of a commercial cotton bed sheet and carbon-coated conductive fabrics with conductive adhesive (Kitagawa Industries, CSTK). The rectangular fabrics having 20 or $25 \mathrm{~mm}$ width were used as lead electrodes and a rectangular fabric with 40 or $50 \mathrm{~mm}$ width was used as a reference electrode. These fabrics were stuck to the bed-sheet with the adhesive in a horizontal-striped pattern at even intervals, as shown in Fig. 3. Convex terminals for lead wire connection were mounted in each fabric on both sides.

Vertical position of the sheet was adjusted so that the reference electrode was placed beneath the breech of the subject lying in a supine position. Two fabrics which were located respectively under the scapulae and the lumbar region were manually selected for the lead electrodes. Capacitive coupling involving skin, sleepwear and electrode was held by the subject's weight on the sleepwear and by repulsive force from the mattress. The electrodes were connected to a measuring device, as described in the next section, by shielded wires. 


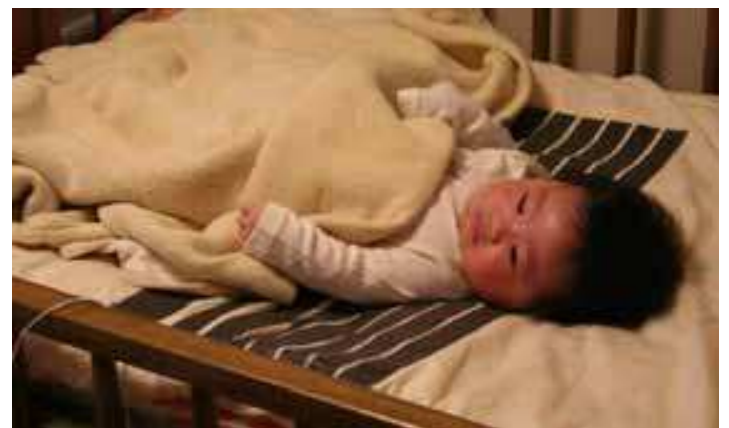

Fig. 3. An image of the subject \#10 lying supine on the electrode unit

\subsection{Pilot Measuring Device}

The pilot measuring device with filtering and amplification circuitry was manufactured according to a block diagram in Fig. 4. The device consisted of a common part and independent parts for sensing ECG signal and breathing activity respectively. The device was powered by regulated batteries to obviate the possibility of electric shock.

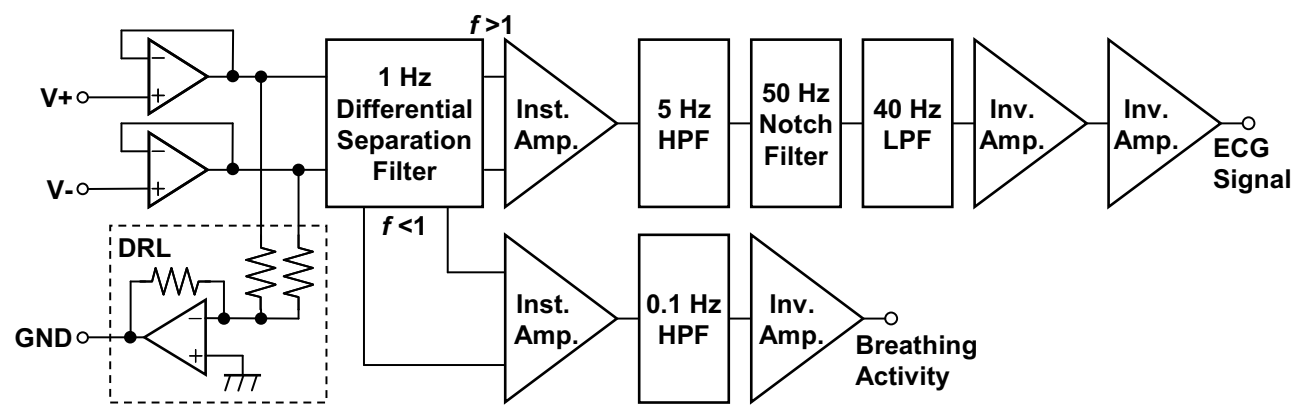

Fig. 4. A block diagram of the developed measuring device

The common part was composed of two buffers, the differential separation filter described in the subsection 2.2 and a driven-right-leg (DRL) circuit. Each buffer functioned as an impedance matching circuit to mediate the high impedance of the capacitive coupling with low impedance required by the subsequent circuitry. Operational amplifier ICs with high input resistance (National Semiconductor, LF356, 1T $\Omega$ according to the specification sheet) were used in the present study. The differential separation filter separated the input signal into high frequency component containing ECG signal $(>1 \mathrm{~Hz})$ and low frequency component including breathing activity $(<1 \mathrm{~Hz})$. The separation filter was constructed of two sets of subtracters, amplifiers and integrators according to Fig. 2. The block diagram in Fig. 2 is a modification of a so called DC suppression circuit (Spinelli et al., 2004). The DRL circuit was employed in order to reduce common mode noise mainly due to power line interference (Spinelli et al., 1999, and Kim et al. 2005). CMRR of the device at 10, 20 and 30 $\mathrm{Hz}$ were 61,61 and $59 \mathrm{~dB}$ respectively.

The independent part for sensing ECG signal consisted of an instrumentation amplifier, a high-pass filter (HPF), a notch filter, a low-pass filter (LPF) and two inverting amplifiers as 
shown in Fig. 4. The circuit elements of the HPF and the LPF were determined in order to obtain a cutoff frequency of 5 and $40 \mathrm{~Hz}$, respectively. The notch filter was used to reduce $50-\mathrm{Hz}$ interference. Although electrocardiograph for diagnostic purpose requires a bandwidth from 0.01 to $100 \mathrm{~Hz}$, we narrowed the bandwidth of the developed device to improve a tolerance for the body motion. Another independent part for obtaining breathing activity consisted of an instrumentation amplifier, a HPF and an inverting amplifier. The HPF was introduced to avoid saturation due to DC offset voltage. Frequency-gain response of the developed device is shown in Fig. 5.

Both output signals from the device were digitized at $1 \mathrm{kHz}$ by an analog-to-digital converter with 16-bit resolution and stored in a personal computer using a data acquisition system (Biopac Systems, MP-150 system). Obtained breathing activity signal was filtered offline with a digital LPF $\left(\mathrm{IIR}, f_{c}=1 \mathrm{~Hz}, \mathrm{Q}=0.707\right)$.

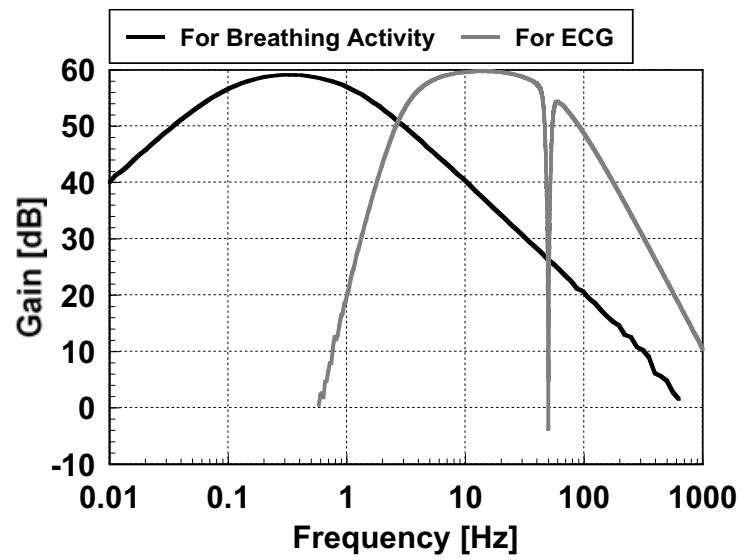

Fig. 5. Frequency-gain responses of the developed circuit for measuring breathing activity and for measuring narrow-band ECG

\subsection{Comparison of Breathing Activity with Respiratory Air Flow in Adult Subjects}

In order to evaluate breathing activity signal obtained with the developed device, simultaneous measurement with respiratory air flow was conducted using a commercial pneumotachograph (Biopac Systems, TSD107). Considering load of wearing a mask on the subject's face, the experiments were conducted for adult subjects instead of infant subjects. Four adult males from whom informed consents were previously obtained participated to the experiment. A bed-sheet electrode unit which we had specified and fabricated for adult subjects in previous study (Ueno et al., 2007b) was employed for the measurement. Each subject was instructed to wear the face mask which was connected to the pneumotachograph and to lie on the bed-sheet electrode unit which was linked with the developed device. As the 1st experiment, the subjects were requested to cease their breathing for about $10 \mathrm{sec}$ after natural breathing. In the 2nd experiment, the subjects were instructed to breathe along to a rhythm of metronome that was set preliminary at a certain speed ranging from 7 to 26 repeat/min. Respiratory rates were calculated respectively from the two signals measured simultaneously with the pneumotachograph and with the developed device. The calculation was conducted automatically using a peak-detection 
function implemented in software (Biopac Systems, Acknowledge 3.9.0) supplied with the data acquisition system. Preparatory filtering with a digital band-pass filter (IIR, 0.1-0.6 Hz, $\mathrm{Q}=0.707)$ was applied off-line to both signals before the calculation.

\subsection{Simultaneous Measurement of Narrow-Band ECG and Breathing Activity in Infants}

Ten infants, aged 53 to 187 days, experienced the experiment (see Table 1). Four of the ten infants partook in the experiment more than once on different age in day. Totally sixteen subjects participated to the experiment. Each subject wearing cotton sleepwear was laid in a supine on the bed-sheet electrode unit. Both high frequency and low frequency components were measured using the developed system from the dorsum of the subject through the sleepwear (and a diaper at the reference electrode). As a reference signal, a directly measured ECG was wirelessly monitored using a commercial bioamplifier (Teac Instruments, BA1104CC) and a commercial telemeter unit (Teac Instruments, TU-4). Two disposable lead electrodes were attached directly to the right and the left flank, and a reference electrode was placed on the frontal surface of the subject's abdomen. To measure another reference signal, a commercial photoplethysmographic sensor (Biopac Systems, TSD200A) was applied to the right or the left earlobe. A special bioamplifier (Biopac Systems, PPG100C) involving a $0.5-100 \mathrm{~Hz}$ band-pass filter (BPF) was used for amplifying the sensor signal. Photoplethysmogram (PPG) was measured only from a part of the subjects because the sensor unit was introduced in the later experiments. The output signals from the developed device (i.e. breathing activity and narrow-band ECG), the reference ECG and the reference PPG were simultaneously measured using the data acquisition system. Preparatory filtering with a digital LPF (IIR, $f_{c}=40 \mathrm{~Hz}, \mathrm{Q}=0.707$ ) was applied to PPG signal to reduce power line interference. Since it is known that breathing activity overlaps with baseline of the PPG (Nakajima et al., 1993), filtering operation using a digital BPF (IIR, 0.1-0.6 Hz) was doubly applied to the preprocessed PPG signal to extract the breathing activity for the third reference signal.

\begin{tabular}{|c|c|c|c|c|c|}
\hline Subject ID & $\begin{array}{c}\text { Age } \\
\text { in day }\end{array}$ & $\begin{array}{l}\text { Weight } \\
{[\mathrm{kg}]}\end{array}$ & Gender & $\begin{array}{c}\text { Thickness of the } \\
\text { clothes }[\mu \mathrm{m}]\end{array}$ & $\begin{array}{c}\text { Reference } \\
\text { signal }\end{array}$ \\
\hline \#1_1st & 65 & 5.4 & male & 1083+diaper & ECG \\
\hline$\# 1$ 2nd & 121 & 6.5 & male & $540+$ diaper & ECG \\
\hline$\# 1 \_3 r d$ & 185 & 7.4 & male & $540+$ diaper & ECG \\
\hline$\# 2$ & 187 & 6.8 & male & $540+$ diaper & ECG \\
\hline \#3_1st & 64 & 4.9 & female & $540+$ diaper & ECG \\
\hline \#3_2nd & 133 & 6.7 & female & $780+$ diaper & ECG \\
\hline \#3_3rd & 167 & 7.0 & female & $680+$ diaper & ECG, PPG \\
\hline \#4_1st & 68 & 7.1 & male & $610+$ diaper & ECG \\
\hline \#4_2nd & 132 & 8.5 & male & $680+$ diaper & ECG, PPG \\
\hline \#5 & 178 & 8.0 & male & 565+diaper & ECG \\
\hline \#6 & 69 & 5.1 & female & $730+$ diaper & ECG \\
\hline \#7_1st & 123 & 7.2 & female & 730+diaper & ECG \\
\hline \#7_2nd & 178 & 7.7 & female & $670+$ diaper & ECG, PPG \\
\hline$\# 8^{-}$ & 130 & 6.2 & female & $570+$ diaper & ECG, PPG \\
\hline \#9 & 53 & 4.5 & female & 707+diaper & ECG, PPG \\
\hline$\# 10$ & 75 & 6.3 & male & $600+$ diaper & ECG, PPG \\
\hline
\end{tabular}

Table 1. Subject information and measured references 


\subsection{Comparison of R-R Intervals using Bland-Altman Plot}

In order to evaluate accuracy of the signal obtained with the developed device, R-R intervals were calculated respectively from the narrow-band ECG signal, the PPG signal and the reference ECG signal, that were measured simultaneously in the subsection 3.4. Each R-R interval was computed automatically using the peak-detection function in the software. Data section with 1-minute length where the triple signals were commonly stable was selected for the analysis for five subjects of \#3_3rd, \#4_2nd, \#8, \#9 and \#10. Preparatory filtering with a digital BPF (IIR, 0.5-40 Hz, Q=0.707) was applied to the all selected data. To develop Bland-Altman Plots (Bland \& Altman, 1986) between the narrow-band ECG and the reference ECG, and also between the PPG and the reference ECG, difference of R-R intervals as well as mean R-R intervals was calculated for corresponding set of R-R intervals in each subject.

\subsection{Comparison of Spectral Powers of Heart Rate Variability}

In order to discuss the effect of detection accuracy of R-R intervals, HR variability (HRV) was analyzed for the triple R-R intervals described in the subsection 3.5. A function of HRV analysis installed in the data acquisition software (Biopac Systems, Acknowledge 3.9.0) was used. Data section of the subject \#9 with 90-sec length where the triple signals were commonly stable was selected for the analysis. Preparatory filtering with a digital BPF (IIR, $0.5-40 \mathrm{~Hz}, \mathrm{Q}=0.707)$ was applied to the selected data. Frequency of HRV from DC to $3 \mathrm{~Hz}$ was analyzed. The spectral power of very low frequency (VLF: DC-0.04 Hz), low frequency (LF: 0.04-0.15 Hz), high frequency (HF: 0.15-0.40 Hz) and very high frequency (VHF: 0.40$3.00 \mathrm{~Hz}$ ) were computed respectively.

\section{Results and Discussions}

\subsection{Comparison of Breathing Activity with Respiratory Air Flow in Adult Subjects}

As can be seen in Fig. 6, output signal from the low frequency part of the developed device (Fig. 6(a)) fairly captured characteristics of air flow signal measured with the commercial pneumotachograph (Fig. 6(b)). We could see a synchronization of both signals in the former part of the recordings and could easily recognize an onset of breath-holding at the beginning of the latter part. These visual synchronization and breath-holding were observed in all 4 subjects. Moreover, respiratory rate calculated from the breathing activities of 4 subjects presented a high correlation $(\mathrm{r}=0.995)$ and was consistent with that calculated from the respiratory air flow, as shown in Fig. 7. Since the chest movements for breathing were observed not only in adults but also in infants in our previous experiments, it is expected that the proposed device is capable of sensing breathing activity in infants with high sensitivity as well. Cause of a subtle error in respiratory rate in Fig. 7 was assumed due to shallow breathing and body motion. Increase in filter order of the HPF or the differential filter in the future is considered to improve the accuracy of the device, because a spectral power from 0.2 to $0.5 \mathrm{~Hz}$, which was mainly originating from motion artifacts, was a certain level in the obtained recordings. 


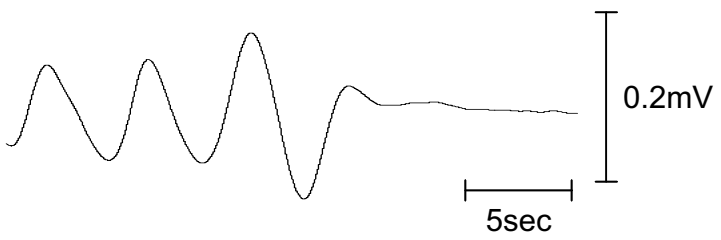

(a) Breathing activity (the developed device)

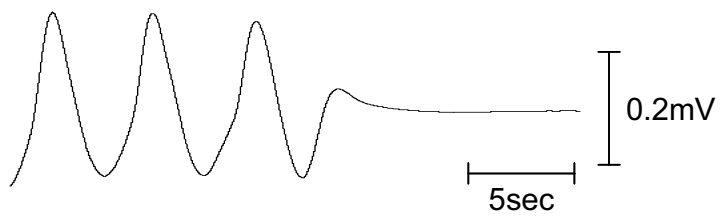

(b) Respiratory air flow (the commercial pneumotachograph)

Fig. 6. Recordings of (a) breathing activity obtained from low frequency part of the developed device, and (b) respiratory air flow measured with the commercial pneumotachograph

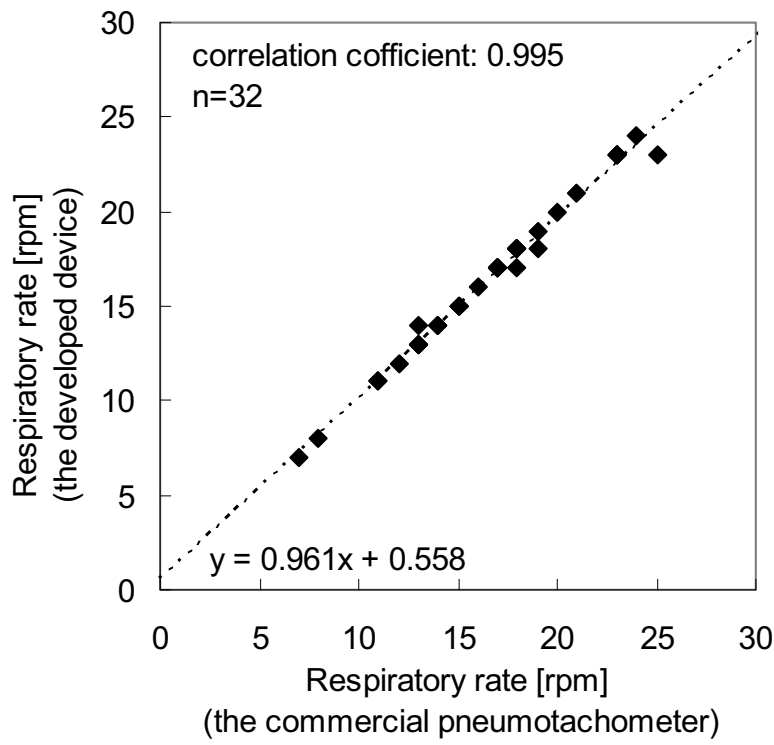

Fig. 7. Correlation of respiration rates between the pneumotachograph and the developed device

4.2 Simultaneous Measurement of Narrow-Band ECG and Breathing Activity in Infants Fig. 8 shows recordings typical of those obtained while subject \#4_2nd was sleeping. Variation of the signal measured with the low frequency part of the developed device (Fig. 
8(a)) was consistent with that of the breathing activity (Fig. 8(b)) derived from the PPG recording in Fig. 8(e). Although a slight phase lag due to mechanical delay of the vessel or to an algorithm of the digital filter was observed in the breathing activity from PPG, this consistency was confirmed in 4 of 6 subjects from whom PPG had been measured as the reference signal. Additionally, synchronizations of sleep-breath sound and signal fluctuation detected with our system were auditory confirmed in 8 of the rest 10 subjects.

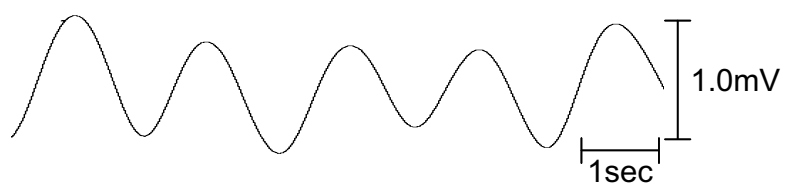

(a) Breathing activity measured with the developed device

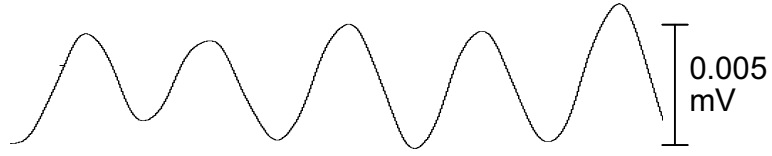

(b) Breathing activity extracted from PPG recording

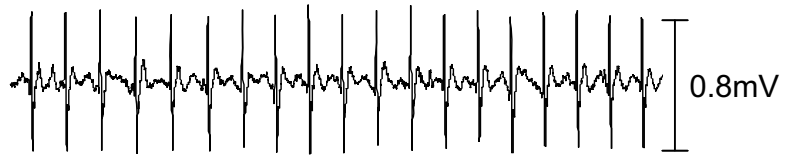

(c) Narrow-band ECG measured with the developed device

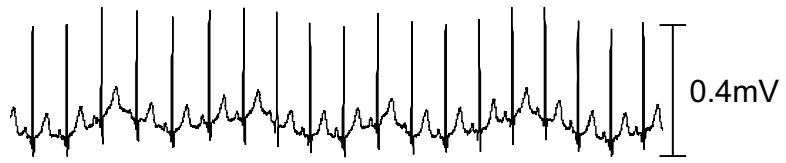

(d) Reference ECG measured with the commercial telemeter

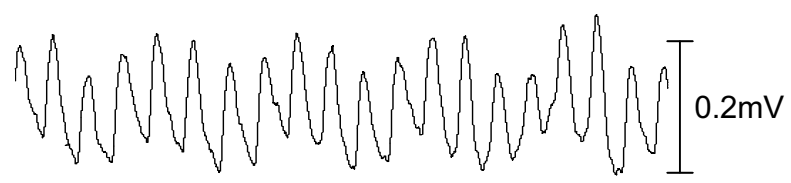

(e) PPG recording measured with the commercial photoplethysmograph

Fig. 8. Typical recordings of (a) breathing activity measured with the low frequency part of the developed device, (b) breathing activity extracted from the PPG recording in Fig. 8(e) using a digital 0.1-0.6 Hz BPF, (c) narrow-band ECG measured with the high frequency part of the developed device, (d) reference ECG measured with the commercial telemeter, (e) PPG recording measured with the commercial photoplethysmograph (subject \#4_2nd)

For the subjects of \#1_1st and \#7_2nd, the developed system failed to measure breathing activity as well as narrow-band ECG because they continued thrashing their limbs with certain intensity throughout the measurement. In the case of subject \#1_2nd and subject \#3_3rd, moderate motions of limbs were generated throughout the measurement, and then 
only breathing activity couldn't be detected. Since time constant of the HPF in the low frequency part (i.e. recovery time against each motion) was longer than that in the high frequency part of the developed device, the moderate motions might lead the failure only for breathing activity measurement in these two subjects. As another cause of the failure, it was considered that the order of HPF was primary. Therefore, shortening of the time constant and increasing of the filter order seemed necessary to improve stability of the system against body motion.

As for heart activity, narrow-band ECG obtained with the high frequency part of the proposed system (Fig. 8(c)) presented periodical spikes synchronized with the reference ECG in Fig. 8(d) and with PPG recording in Fig. 8(e). The synchronization with at least one reference was observed in 14 of 16 subjects. In the subjects of \#1_1st and \#7_2nd, stable output signals couldn't be measured for the reason noted above.

These results demonstrate that the proposed system is capable of sensing breathing activity and narrow-band ECG simultaneously whenever the subject is sleeping or in a resting state even wearing a diaper and a sleepwear. Although there is still room for improvement in terms of its practical use, the proposed system appears promising for application to infant monitor to sense breathing activity and HR without attaching any sensors directly on their skins. As for susceptibility of the system to body motion, there are considered two ways of approaching. One is the improvement of stability of the system against the body motion, as previously stated. Another is active utilization of the susceptibility for detection of defective arousal reaction in infants. The defective arousal reaction has been implicated in the development of SIDS (Sawaguchi \& Tedsuka, 1999). Franco et al. reported that risk factors such as "exposure to cigarette smoking" and "prone position" decreased arousals in infants (Franco, 1998, 1999, 2004, and Groswasser, 2001). Therefore alternative use of the low frequency part of the developed device for detecting breathing activity or body motion caused by defective arousal reaction would be beneficial for a preventive SIDS monitor.

\subsection{Comparison of R-R Intervals using Bland-Altman Plot}

It is known that scattering plots in Bland-Altman plot with small dependency on horizontal axis and with small vertical deviation from the zero line indicate preciseness of the method under consideration compared with another referencing method. As can be seen, distribution of plots in Fig. 9(a) was independent of the horizontal axis and was obviously narrower along with a horizontal zero line than that in Fig. 9(b). Corresponding to the distributions, 95\% confidence interval between the reference ECG and narrow-band ECG in Fig. 9(a) was more than 30 times smaller than that between the reference ECG and PPG in Fig. 9(b). Since accuracy of R-R interval in narrow-band ECG was within $\pm 2 \mathrm{~ms}$ and more than 30 times higher than that in PPG, the developed device can be used not only for monitoring ECG but also for measuring precise HR instantaneously. 


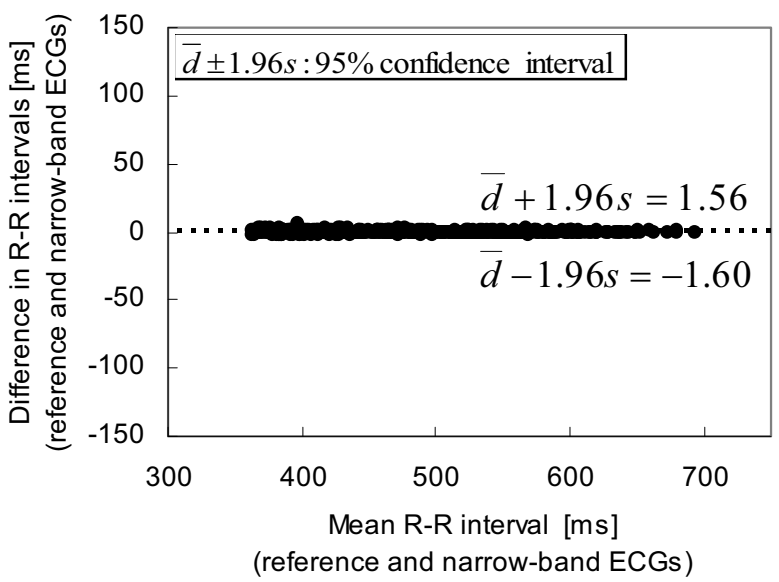

(a) between reference ECG and narrow-band ECG

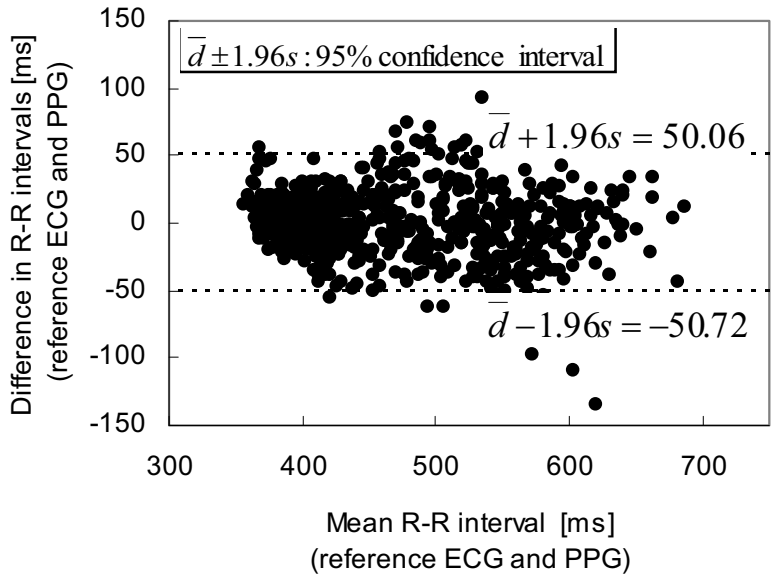

(b) between reference ECG and PPG

Fig. 9. Comparison of R-R intervals using Bland-Altman plot among the reference ECG, narrow-band ECG measured the developed device and PPG

\subsection{Comparison of Spectral Powers of Heart Rate Variability}

High accuracy of HR calculated from narrow-band ECG can be recognized also in Table 2. In accordance with the results of Fig. 9, powers of HRV in the frequency bands of VLF, LF and HF that were analyzed from narrow-band ECG were identical respectively to those from the reference ECG. In contrast, the powers obtained from the PPG contained errors more than $0.50 \%$ in above all frequency bands even though a short-time data section with 90 sec length was used for the analysis. In addition, the power in VHF band obtained from 
the narrow-band ECG was 100 times more accurate than that from the PPG. Thus, the results in Table 2 also support that the developed device has an advantage in measuring accurate HR for a long time.

This advantage of our system may be utilized in other applications, for instance, in detecting seizures in the newborn. Greene (Greene et al., 2007) proposed a method for the detection of seizures in the newborn using heartbeat timing interval features. Seizures occur in 6-13\% of low birth weight infants and 1-2 per 1000 infants born at term (Rennie, 1997). Clinical evidence suggests that neonates with seizures have poor health outcomes, with morbidity in $50 \%$ of survivors, and a high (30\%) probability of death (Tharp, 2002). Therefore, it seems a critical issue to increase performance of our system so as to be utilized in NICU (neonatal intensive care unit). Another example is an application to adult (particularly elderly) subjects. Since it is reported that autonomic nervous system dysfunction, estimated by high HR and low HRV, may be associated with the development of diabetes in healthy adults (Carnethon, 2003), our system would be suitable for an awareness-free HR monitor in daily use for health management.

\begin{tabular}{cccc}
\hline \multirow{2}{*}{ Frequency band [Hz] } & \multicolumn{3}{c}{ Power of HRV [s $\left.{ }^{2}\right]$} \\
\cline { 2 - 4 } & Reference ECG & PPG & Narrow-band ECG \\
\hline VLF: DC-0.04 & 0.118 & $0.117(0.78 \%)$ & $0.118(0.00 \%)$ \\
LF: $0.04-0.15$ & 0.858 & $0.853(0.50 \%)$ & $0.858(0.00 \%)$ \\
HF: $0.15-0.40$ & 0.432 & $0.435(0.71 \%)$ & $0.432(0.00 \%)$ \\
VHF: $0.40-3.00$ & 0.235 & $0.221(5.75 \%)$ & $0.234(0.05 \%)$ \\
\hline
\end{tabular}

*Values in ( ) indicate error rate against the power obtained from the reference ECG

Table 2. Comparison of spectrum powers of HRV among the reference ECG, the reference PPG, and the narrow-band ECG measured with the developed device

\section{Conclusion and Future Prospects}

We proposed an approach for obtaining narrow-band ECG as well as breathing activity simultaneously from an infant who wears a diaper and a sleepwear, and lies supine on a bed-sheet electrode unit. We manufactured a pilot measuring device based on the approach and performed verification experiments for 4 adults and for 16 infant subjects. The measurement yielded the following results.

- We could see an onset of breath-holding and synchronized variations in breathing activity signal measured with the low frequency part of the developed device for 4 adult subjects, compared with respiratory air flow signal measured with a commercial pneumotachograph.

- Respiratory rate calculated from the breathing activity was highly correlated $(\mathrm{r}=0.995)$ and consistent with that calculated from the respiratory air flow.

- The proposed system was capable of sensing breathing activity and narrow-band ECG simultaneously whenever the infant subject was sleeping or in a resting state even wearing a diaper and a sleepwear.

- Since accuracy of R-R interval in narrow-band ECG was within $\pm 2 \mathrm{~ms}$ and more than 30 times higher than that in PPG, the developed device can be used not only for monitoring ECG but also for measuring precise HR. 
- High accuracy of HR calculated from narrow-band ECG could be recognized also in the spectral powers of HRV.

Although the system is susceptible to body motions when the subject is alive and thus there is still room for improvement in terms of its practical use, the proposed system appears promising for application to infant monitor to sense breathing activity and accurate HR without attaching any sensors directly on their skins.

Future issues to be addressed are as follows: (1) improvement of stability of the system against body motions, (2) combination with software for detecting life threatening events of infants, (3) bandwidth extension of the part for ECG measurement so as to derive time domain parameters such as QT interval, (4) application to aged subjects with a view to use in home healthcare.

\section{Acknowledgment}

This study was supported in part by Academic Frontier Project for Private Universities: matching fund subsidy from MEXT (Ministry of Education, Culture, Sports, Science and Technology), 2003-2004, in part by Industrial Technology Research Grant Program in 20052008 from NEDO (New Energy and Industrial Technology Development Organization of Japan), and in part by Grant-in-Aid for Young Scientists (B) in 2009 (21 700512).

\section{References}

Asaishi, T.; Ueno, A.; Hoshino, H.; Mitani, H. \& Ishiyama, Y. (2002). Measurement of sympathetic skin response by using DC servo circuit -Detection of DC and AC components, and an application for estimating the nerve's conduction velocity-, Life Support, Vol. 14, No. 3, 10-15, 1341-9455

Bland, J.M. \& Altman, D.G. (1986). Statistical methods for assessing agreement between two methods of clinical measurement, The Lancet, Vol.1, 307-310, 0140-6736

Carnethon, M.R.; Golden, S.H.; Folsom, A.R.; Haskell, W. \& Liao, D. (2003). Prospective investigation of autonomic nervous system function and the development of type 2 diabetes: The atherosclerosis risk in communities study 1987-1998, American Heart Journal, Vol. 107, 2190-2195, 0002 - 8703

Catrysse, M.; Puers, R.; Hertleer, C.; Van Langenhove, L.; Van Egmondc, H. \& Matthys, D. (2004). Towards the integration of textile sensors in a wireless monitoring suit, Sensors and Actuators A, Vol. 114, 302-311, 0924-4247

Franco, P.; Pardou, A.; Hassid. S.; Lurquin, P.; Groswasser, J. \& Kahn, A. (1998). Auditory arousal thresholds are higher when infants sleep in the prone position, Journal of Pediatrics, Vol. 132, 240-243, 00223476

Franco, P.; Groswasser, J.; Hassid, S.; Lanquart, J.P.; Scaillet, S. \& Kahn, A. (1999). Prenatal exposure to cigarette smoking is associated with a decrease in arousal in infants, Journal of Pediatrics, Vol. 135, 34-38, 00223476

Franco, P.; Seret, N.; Van Hees, J.N.; Scaillet, S.; Vermeulen, F.; Groswasser, J. \& Kahn, A. (2004). Decreased arousals among healthy infants after short-term sleep deprivation, Pediatrics, Vol. 114, 192-197, 0031-4005 
Furusawa, Y.; Ueno, A.; Hoshino, H.; Kataoka, S.; Mitani H. \& Ishiyama, Y. (2003). Low invasive measurement of electrocardiogram for newborns and infants, Proceedings CD-ROM of the IEEE EMBS Asian-Pacific Conference on Biomedical Engineering, No.022216-1, Keihanna Plaza Hotel, 0-7803-7944-6, October 2003, IEEE Publishing, Piscataway

Gramse, V.; De Groote, A. \& Paiva, M. (2003). Novel concept for a noninvasive cardiopulmonary monitor for infants -A pair of pajamas with an integrated sensor module, Annals of Biomedical Engineering, Vol. 31, 152-158, 0090-6964

Greene, B.R.; de Chazal, P.; Boylan, G.B.; Connolly, S. \& Reilly, R.B. (2007). Electrocardiogram based neonatal seizure detection, IEEE Transactions on Biomedical Engineering, Vol.54, No.4, 673-682, 0018-9294

Groswasser, J.; Simon, T.; Scaillet, S.; Franco, P. \& Kahn, A. (2001). Reduced arousals following obstructive apneas in infants sleeping prone, Pediatric Research, Vol.49, 402-406, 00313998

Kato, T.; Ueno, A.; Kataoka, S.; Hoshino, H. \& Ishiyama, Y. (2006). An application of capacitive electrode for detecting electrocardiogram of neonates and infants, Proceedings of 28th Annual International Conference of the IEEE EMBS, pp. 916-919, 14244-0033-3, Marriott at Times Square, Sept. 2006, IEEE Publishing, Piscataway

Kim, K.K.; Lim, Y.K. \& Park, K.S. (2005). Common mode noise cancellation for electrically non-contact ECG measurement system on a chair, Proceedings of 27th Annual International Conference of the IEEE EMBS, pp. 5881-5883, 0-7803-8740-6, Shanghai International Convention Center, Sept. 2005, IEEE Publishing, Piscataway

Krous, H.F.; Beckwith, J.B.; Byard, R.W.; Rognum, T.O.; Bajanowski, T.; Corey, T.; Cutz, E.; Hanzlick, R.; Keens, T.G. \& Mitchell, E.A. (2004). Sudden infant death syndrome and unclassified sudden infant deaths: A definitional and diagnostic approach, Pediatrics, Vol. 114, No. 1, 234-238, 1098-4275

Little, G.A.; Ballard, R.A.; Brooks, JG et al. (1987). National Institutes of Health consensus development conference on infantile apnea and home monitoring, Sept 29 to Oct 1 , 1986, Pediatrics, Vol. 79, No. 2, pp. 292-299, 0031-4005

Lopez, J.A. \& Richardson, P.C. (1969). Capacitive electrocardiographic and bioelectric electrodes, IEEE Transactions on Biomedical Engineering, Vol.BME-16, 99, 0018-9294

Nakajima, K.; Tamura, T. \& Miike, H. (1993). Heart and respiratory rates monitor using digital filters. Japanese Journal of Medical Electronics and Biological Engineering, Vol. 31, No. 4, 360-366, 00213292

Richardson, P. C.; Coombs, F.K. \& Adams, R.M. (1968). Some new electrode techniques for long term physiologic monitoring, Aerospace Medicine, Vol.39, 745-750, 0001-9402

Pallàs-Areny, R. \& Webster, J.G. (1999). 7.1.6 Differential Filters, In: Analog Signal Processing, 333-337, John Wiley \& Sons, 978-0471125280, U.S.A.

Rennie, J.M. (1997). Neonatal seizures, European Journal of Pediatrics, Vol. 156, 83-87, 14321076

Sawaguchi, T. \& Tedsuka, Y. (1999). The physiological definition of the arousal reaction in infants in reference to the hypothesis of defective arousal reaction in SIDS, Research and Practice in Forensic Medicine, Vol. 42, 341-346, 0289-0755

Spinelli, E.M.; Martínez, N.H. \& Mayosky, M.A. (1999). A transconductance driven-right-leg circuit, IEEE Transactions on Biomedical Engineering, Vol. 46, No. 12, 1466-1470, 00189294 
Spinelli, E.M.; Martínez, N; Mayosky, M.A. \& Pallàs-Areny, R. (2004). A novel fully differential biopotential amplifier with DC suppression, IEEE Transactions on Biomedical Engineering, Vol. 51, No. 8, 1444-1448, 0018-9294

Statistics and Information Department, Ministry of Health, Labour and Welfare, Japan (2007). Vital Statistics Japan, http://www.mhlw.go.jp/toukei/saikin/hw/jinkou/ suii07/deth8.html

Tharp, B.R. (2002). Neonatal seizures and syndromes. Epilepsia, Vol. 43, 2-10, 0013-9580

Ueno, A.; Furusawa, Y.; Hoshino, H. \& Ishiyama, Y. (2004). Detection of electrocardiogram by electrodes with fabrics using capacitive coupling, IEEJ Transactions on Electronics, Information and Systems, Vol. 124, No. 9, 1664-1671, 0385-4221

Ueno, A.; Akabane, Y.; Kato, T.; Hoshino, H.; Kataoka, S. \& Ishiyama, Y. (2007a). Capacitive sensing of electrocardiographic potential through cloth from the dorsal surface of the body in a supine position -A preliminary study, IEEE Transactions on Biomedical Engineering, Vol. 54, No. 4, 759-766, 0018-9294

Ueno, A.; Shiogai, Y. \& Ishiyama, Y. (2007b). A primary study of indirect ECG monitor embedded in a bed for Home Health Care, IEEJ Transactions on Electronics, Information and Systems, Vol. 127, No. 10, 1792-1799, 0385-4221

Ueno, A. \& Yama, Y. (2008). Unconstrained monitoring of ECG and respiratory variation in infants with underwear during sleep using a bed-sheet electrode unit, Proceedings of 30th Annual International Conference of the IEEE EMBS, pp. 2329-2332, 978-1-42441815-2, Vancouver Convention \& Exhibition Centre, Aug. 2008, IEEE Publishing, Piscataway

Yama, Y. \& Ueno, A. (2009). Unrestrained facile measurement of narrow-band ECG and respiratory variation in infants with a capacitive sheet-type sensor, Transactions of Japanese Society for Medical and Biological Engineering, Vol. 47, No. 1, 42-50, 1347-443X 


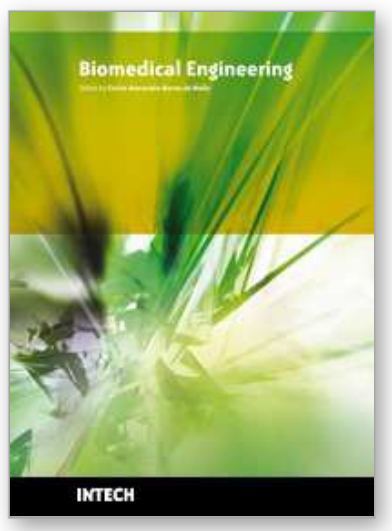

\author{
Biomedical Engineering \\ Edited by Carlos Alexandre Barros de Mello
}

ISBN 978-953-307-013-1

Hard cover, 658 pages

Publisher InTech

Published online 01, October, 2009

Published in print edition October, 2009

Biomedical Engineering can be seen as a mix of Medicine, Engineering and Science. In fact, this is a natural connection, as the most complicated engineering masterpiece is the human body. And it is exactly to help our "body machine" that Biomedical Engineering has its niche. This book brings the state-of-the-art of some of the most important current research related to Biomedical Engineering. I am very honored to be editing such a valuable book, which has contributions of a selected group of researchers describing the best of their work. Through its 36 chapters, the reader will have access to works related to ECG, image processing, sensors, artificial intelligence, and several other exciting fields.

\title{
How to reference
}

In order to correctly reference this scholarly work, feel free to copy and paste the following:

Akinori Ueno, Tatsuya Imai, Daisuke Kowada and Yoshihiro Yama (2009). Capacitive Sensing of Narrow-Band ECG and Breathing Activity of Infants through Sleepwear, Biomedical Engineering, Carlos Alexandre Barros de Mello (Ed.), ISBN: 978-953-307-013-1, InTech, Available from: http://www.intechopen.com/books/biomedicalengineering/capacitive-sensing-of-narrow-band-ecg-and-breathing-activity-of-infants-through-sleepwear

\section{INTECH}

open science | open minds

\section{InTech Europe}

University Campus STeP Ri

Slavka Krautzeka 83/A

51000 Rijeka, Croatia

Phone: +385 (51) 770447

Fax: +385 (51) 686166

www.intechopen.com

\section{InTech China}

Unit 405, Office Block, Hotel Equatorial Shanghai

No.65, Yan An Road (West), Shanghai, 200040, China

中国上海市延安西路65号上海国际贵都大饭店办公楼 405 单元

Phone: +86-21-62489820

Fax: $+86-21-62489821$ 
(C) 2009 The Author(s). Licensee IntechOpen. This chapter is distributed under the terms of the Creative Commons Attribution-NonCommercial-ShareAlike-3.0 License, which permits use, distribution and reproduction for non-commercial purposes, provided the original is properly cited and derivative works building on this content are distributed under the same license. 